\title{
Química Verde no desenvolvimento de materiais de afinidade para purificação de princípios ativos farmacêuticos
}

\author{
Raquel Viveiros \\ CleanMIPTech group, LAQV-REQUIMTE, Departamento de Química, Faculdade de Ciências e Tecnologia, \\ Universidade NOVA de Lisboa, 2829-516 Caparica, Portugal \\ rfv17327@campus.fct.unl.pt
}

\begin{abstract}
Green development of cutting-edge affinity materials to address API purification challenges - Pharmaceutical industry spends a very significant amount of its financial resources in API (Active Pharmaceutical Ingredients) purification processes to comply with impurity limits imposed by regulatory agencies (FDA and EMEA). To address this worldwide demand, several purification materials have been proposed, such as molecular imprinted polymers (MIPs). These cutting-edge affinity materials can be produced by different conventional approaches. In the last years, the use of the Green Chemistry principles has changed the way polymers can be produced. Green technologies applied to MIP development are appearing not only due to environmental issues but also by the new material's properties and cost-effective production from the point of view of industry. MIPs have been developed in our lab using supercritical carbon dioxide $\left(\mathrm{scCO}_{2}\right)$ technology. These affinity materials have been developed for a wide range of applications, namely pharmaceutical impurity removal, enrichment of natural products, removal of contaminants from diesel and from water resources. They are obtained as ready-to-use and easy-to-handle dry-powders of homogenous particle sizes, without organic solvent residues.
\end{abstract}

A indústria farmacêutica despende uma quantidade muito significativa de seus recursos financeiros em processos de purificação de APIs (Princípios Ativos Farmacêuticos) para cumprir os limites de impurezas impostas pelas agências reguladoras (FDA e EMEA). Para responder a esta demanda mundial, têm sido propostos vários materiais, como os polímeros molecularmente impressos (MIPs). Esses materiais de afinidade do tipo "chave-fechadura" são produzidos usando diferentes metodologias convencionais. O uso dos princípios da Química Verde tem mudado a forma como os polímeros são produzidos. As tecnologias verdes aplicadas ao desenvolvimento de MIPs apareceram não só devido às preocupações com o meio ambiente, mas também devido às características do produto final e a sua produção económica. Os MIPs têm sido desenvolvidos no laboratório usando a tecnologia de dióxido de carbono supercrítico $\left(\mathrm{scCO}_{2}\right)$. Estes materiais de afinidade já foram desenvolvidos para uma vasta gama de aplicações, desde a remoção de impurezas farmacêuticas até ao enriquecimento de produtos naturais. Os materiais de afinidade do tipo "chave-fechadura” são obtidos prontos a usar, na forma de pós secos, fáceis de manusear, com distribuição de tamanhos de partícula homogénea e sem resíduos de solventes orgânicos.

\section{Imitando a Natureza}

Desde sempre que tentamos imitar a natureza, nomeadamente nos processos de afinidade entre moléculas como, por exemplo, a interação entre anticorpos e antigénios. Um dos maiores desafios dos cientistas é tentar perceber e reproduzir quimicamente o mecanismo por detrás destes processos de afinidade.

O processo de afinidade é um processo de reconhecimento molecular em que os polímeros molecularmente impressos (MIPs, de molecular imprinted polymers) mimetizam o reconhecimento molecular à semelhança de um anticorpo natural, criando sítios específicos, que são cavidades, em matrizes poliméricas reticuladas para uma determinada molécula (template) à qual se pretende ter afinidade, à semelhança do antigénio [1]. Estas cavidades são complementares quer em funcionalidade química quer em tamanho e forma para o template. Os MIPs têm encontrado aplicações em várias áreas, desde processos de separação/ purificação, sensores, catálise e libertação controlada de fármacos. São também uma alternativa ao uso de biomoléculas, que tendem a perder as suas propriedades de reconhecimento (desnaturar) em ambientes extremos e têm um elevado custo, o que limita a sua utilização.
O processo de síntese de MIPs (Fig. 1) envolve uma reação de polimerização reticulada na presença do template. Numa primeira fase forma-se um complexo estável entre cada molécula de template e os monómeros funcionais. Em seguida, este complexo é imobilizado através da copolimerização com um agente reticulante. O processo ocorre na presença de um solvente porogénico, que tem extrema influência no processo, pois dita o tipo de interações estabelecidas no meio, tendo também impacto na morfologia do polímero, na acessibilidade às cavidades e também no seu desempenho. No final da reação o template é extraído do MIP deixando os sítios ativos vazios e prontos a usar numa posterior aplicação.

Existem vários métodos de síntese de MIPs. Contudo estes métodos apresentam algumas limitações, nomeadamente a necessidade de se utilizar grandes quantidades de solventes orgânicos nestas sínteses e o facto de os polímeros serem obtidos tipicamente em forma de bloco, o que faz com que sejam necessários processos de secagem/moagem e o uso de peneiros para separar as frações do polímero. O processo envolvido nestas vias de síntese é muito moroso e, além disso, compromete a homogeneidade dos sítios ativos de ligação gerados durante o processo de síntese do MIP. Por outro lado, os MIPs são muito atrativos pois 


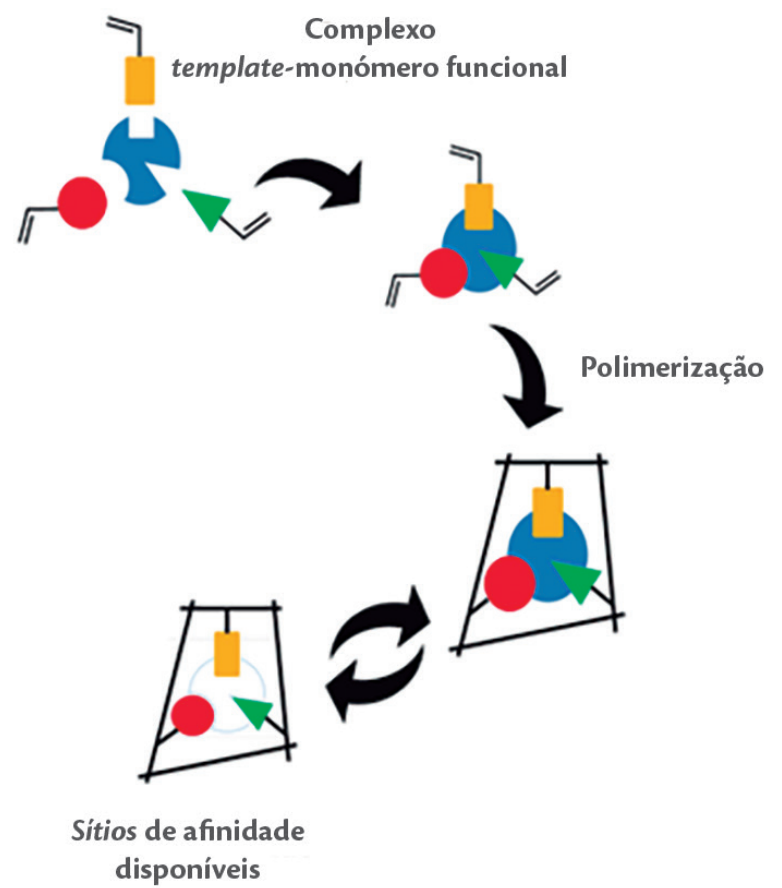

Figura 1 - Esquema geral do processo de impressão molecular, compreendendo: formação do complexo template-monómero funcional, seguindo da polimerização dos monómeros funcionais, em que é obtido um polímero reticulado, e a desorção do template da matriz.

apresentam constantes de ligação semelhantes aos recetores naturais [2,3], são resistentes a condições extremas de pressão, temperatura e $\mathrm{pH}$, podem ser utilizados nos mais variados solventes orgânicos, têm um processo de síntese muito económica, facilmente escalável e com boa reprodutibilidade. Os MIPs produzidos em meios convencionais têm sido desenvolvidos para inúmeras aplicações desde extração de fase sólida, cromatografia e membranas, entre outros. Contudo, as crescentes restrições ao uso de solventes orgânicos voláteis têm levado os cientistas a procurar novos métodos, mais limpos e amigos do ambiente, para a produção de materiais de afinidade mais sustentáveis, usando água, dióxido de carbono supercrítico $\left(\mathrm{scCO}_{2}\right)$, micro-ondas, líquidos iónicos, ultrassons, eletropolimerização e métodos computacionais [4].

\section{Porquê purificar os princípios ativos farmacêuticos?}

Na síntese de APIs são usados reagentes que induzem o aparecimento de intermediários e subprodutos. Apesar do processo de processamento intensivo, no último estágio da produção ainda existem impurezas no material obtido. Por isso, a remoção de impurezas dos APIs é uma questão muito importante em processos de produção farmacêutica e, mais ainda, se essas impurezas forem potencialmente genotóxicas.

Durante muitos anos não houve qualquer norma específica que regulamentasse o conteúdo de impurezas nos APIs [5]. Só em 2006 é que as entidades reguladoras impuseram limites na quantidade de compostos genotóxicos presentes nos fármacos [9]. Os avanços tecnológicos observados nas técnicas analíticas vieram permitir rastrear quantidades muito pequenas de impurezas que dificilmente eram detetadas anteriormente. Esta situação levou a indústria farmacêutica a procurar processos de purificação mais efi- cientes para o desenvolvimento e produção de APIs, que consomem muito tempo e recursos económicos, tornando urgente a necessidade de encontrar novas soluções de purificação que sejam seletivas, por exemplo com base na estrutura molecular.

Tipicamente, na síntese de APIs são usados solventes orgânicos até ao final do processo onde os produtos com elevado valor acrescentado são separados de misturas complexas usando processos como a extração, a cristalização, a precipitação e a destilação. Embora estes métodos já estejam implementados, conduzem a perdas elevadas do API (até 20\%) [8], o que faz com que o custo final do produto aumente significativamente. Consequentemente, são bem-vindos novos processos ou materiais tecnológicos altamente seletivos, que sejam mais amigos do meio ambiente, com menos etapas de processo e também menos morosos, e que tenham um consumo de energia mais reduzido, para atender à necessidade farmacêutica mundial.

Engenharia Verde na procura de soluções mais sustentáveis para a purificação de princípios ativos farmacêuticos

$\mathrm{O}$ scCO $\mathrm{C}_{2}$ um solvente "verde" que reúne propriedades muito interessantes do ponto de vista económico. É o fluido supercrítico mais utilizado. Tem um ponto crítico baixo (31 ${ }^{\circ} \mathrm{C}$ e 73 bar), e é um gás nas condições de temperatura e pressão normais, o que significa que pode ser libertado facilmente para a atmosfera.

$\mathrm{O} \mathrm{scCO}_{2}$ é um excelente meio para sintetizar e processar polímeros ou outras estruturas [9], para a impregnação de fármacos [14] e para a produção de partículas [16]. É uma alternativa viável e sustentável no desenvolvimento de MIPs [4], trazendo benefícios quer na formação dos sítios específicos quer na forma como o polímero é obtido, tipicamente sob a forma de pó seco com morfologia controlada, isento de solventes e sem necessidade de passos de purificação adicionais. Os MIPs assim obtidos apresentam um bom desempenho não só em solventes orgânicos como também em meios aquosos, o que permite a sua potencial aplicação em fluidos biológicos, por exemplo. O processo de extração do template ocorre também em $\mathrm{scCO}_{2}$ pois este aumenta até 10 vezes o coeficiente de difusão do template da matriz comparativamente aos métodos de extração convencionais [17].

A purificação de APIs é um passo crucial no processo de produção de API pois deve garantir as especificações de qualidade do medicamento. É sabido que 50-80\% dos custos de produção correspondem a processos de purificação. Além disso, os métodos atuais são complexos, requerem processos combinados, caros e intensivos para alcançar os níveis de qualidade exigidos.

Desde 2013, têm vindo a ser desenvolvidos diversas técnicas e materiais de adsorção de elevada afinidade para a remoção de genotoxinas, formadas durante o processo de produção de APIs, baseados na tecnologia de impressão molecular em $\mathrm{scCO}_{2}$, tais como: a) partículas poliméricas MIP; b) partículas suportadas por MIP para funcionarem como dispositivos gravitacionais; e c) desenho racional de MIPs usando SYBYL - um programa computacional que introduz o $\mathrm{CO}_{2}$ como solvente na simulação e que permite encontrar uma maneira económica de otimizar a síntese de polímeros de afinidade em $\mathrm{scCO}_{2}$. 
Numa primeira abordagem foram produzidas partículas poliméricas MIP (Fig. 2) para funcionarem como empacotamento de dispositivos de afinidade aplicados a processos de produção de APIs. Foram usados a acetamida (ACET) (uma impureza farmacêutica modelo), o ácido metacrílico (MAA) e metacrilamida (MAM) como monómeros e dimetacrilato de etilenoglicol (EGDMA) como reticulante para a produção de MIPs em $\mathrm{scCO}_{2}$. As partículas foram obtidas com uma distribuição de tamanho de partícula estreita e foram capazes de remover pelo menos 1,98 mmol de ACET por grama de polímero enquanto que em estudos descritos na literatura só conseguiram remover 0,084 mmol de ACET por grama de polímero [18], mostrando que esta é uma estratégia sustentável e mais eficiente do que as reportadas até então.

a)

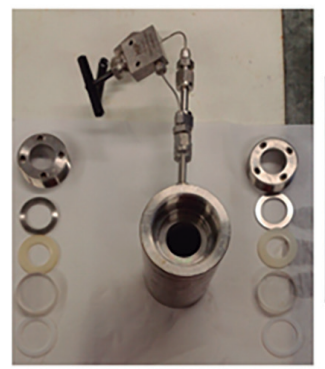

b)

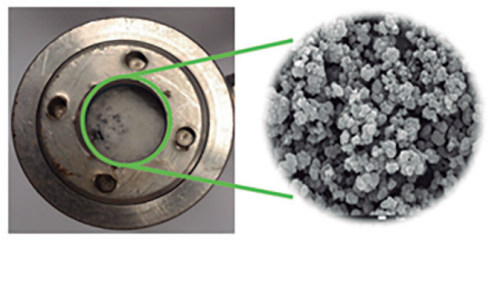

Figura 2 - a) Reator típico usado para produzir MIPs e b) Aparência de polímeros produzidos em $\mathrm{scCO}_{2}$ cujo tamanho médio de partícula varia entre 3,4 e $5,3 \mu \mathrm{m}$.
Uma segunda abordagem, desenvolvida em colaboração com a Hovione [19,20], envolveu a produção de partículas de grandes dimensões com uma camada de MIP na sua superfície para remoção de impurezas na produção de APIs. As partículas com uma camada de MIP à superfície foram previamente funcionalizadas usando dois métodos diferentes, grafting to (ligação para) e grafting from (ligação a partir de), usando, respetivamente, um agente silano de ligação - (metacrilato de 3-(trimetoxisilil)propilo)/etanol$\mathrm{scCO}_{2}$ e tecnologia de plasma (E1: árgon e E2: árgon + hidrogénio) (Fig. 3). A camada MIP, com afinidade para a ACET, foi produzida na superfície da sílica usando MAA como monómero e EGDMA como reticulante.

Para a avaliação do desempenho do protótipo gravitacional foi usada uma mistura modelo contendo a impureza e dipropionato de beclometasona, ACET e API respetivamente, em que as partículas produzidas usando as condições de plasma E2 demonstraram ter o melhor desempenho, removendo $100 \%$ de ACET com uma perda de 0,32\% de API (Fig. 4). Esta estratégia gerou resultados muito promissores não só devido ao facto da coluna funcionar em modo gravitacional, mas também em termos do equilíbrio da remoção de ACET vs API, mostrando ser uma maneira eficiente de remover a ACET de uma mistura farmacêutica. Os processos tipicamente utilizados requerem sistemas de pressão ou de vácuo acoplados e as perdas de API são superiores ( 24\%) aos valores obtidos com esta estratégia (0,32\%).

Numa perspetiva de se obter um melhor desempenho dos materiais de afinidade, foi explorada uma abordagem

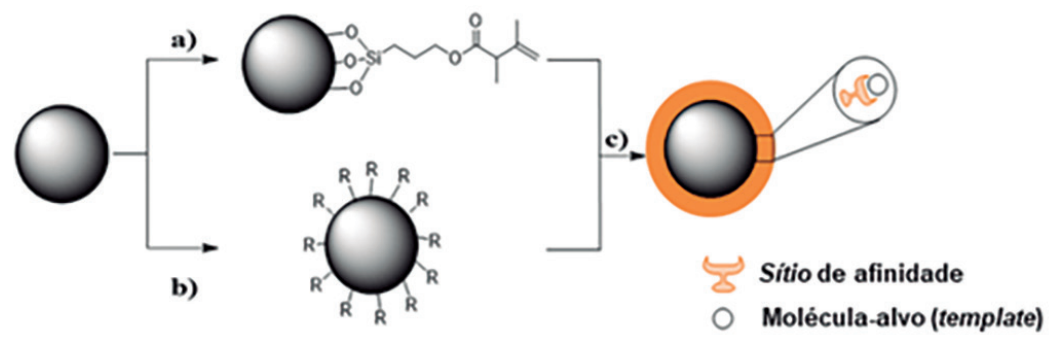

Figura 3 - Esquema geral para a produção de partículas de grandes dimensões (75-200 $\mu \mathrm{m})$ com uma camada de MIP à superfície: a) Funcionalização das partículas com um agente de ligação silano - MPS em $\mathrm{scCO}_{2}$, b) Funcionalização através da tecnologia de plasma ( $R$ são os radicais produzidos na superfície das partículas) sob as condições E1 e E2, c) Produção de uma camada MIP de partículas na superfície das partículas esféricas de grandes dimensões. Adaptada da referência [19] com permissão da Elsevier.

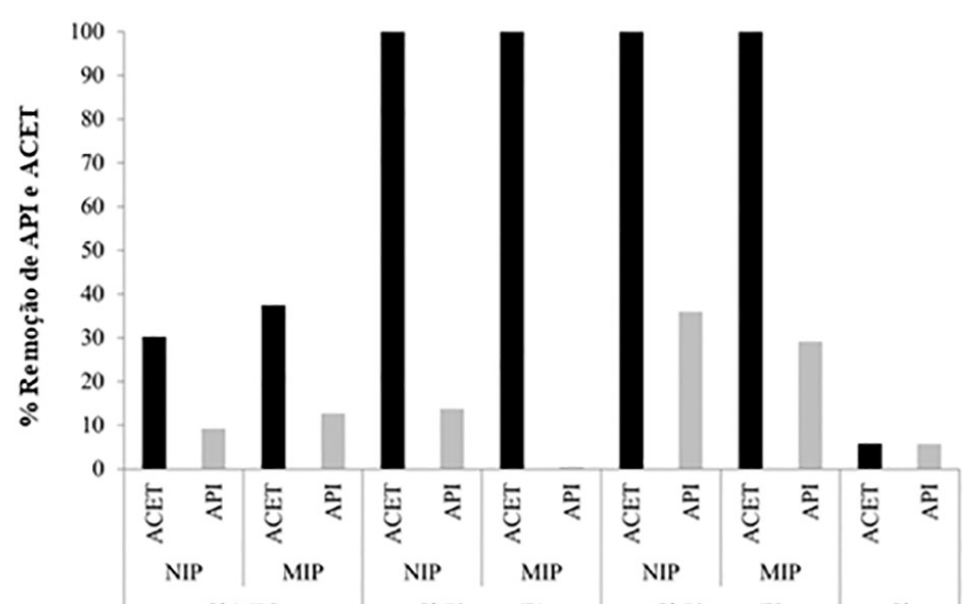

Figura 4 - Percentagem de remoção seletiva da ACET e API, pelas partículas revestidas com uma camada de MIP. Reproduzida da referência [19] com permissão da Elsevier. 
combinada computacional + experimental que envolveu o uso do programa computacional SYBYL ${ }^{\mathrm{TM}}$ para o desenho racional de MIPs para ACET, onde o $\mathrm{CO}_{2}$ foi introduzido pela primeira vez como solvente na simulação [10]. O SYBYL ${ }^{\mathrm{TM}}$, para além de permitir desenhar computacionalmente um MIP 3D para o template, ou seja, o complexo template:monómero funcional, consegue também indicar qual o melhor monómero para aquele template específico, bem como a proporção template:monómero funcional que irá gerar o melhor desempenho do material de afinidade. Para isso é necessário introduzir no programa uma base de dados que contenha todos os monómeros polimerizáveis. A partir da base de dados, o programa computacional conseguiu gerar um ranking dos monómeros mais adequados, bem como as melhores composições molares a serem utilizadas experimentalmente na síntese do MIP em $\mathrm{scCO}_{2}$. Foi selecionado o ácido itacónico (ITA) e o metacrilato de 2-hidroxietilo (HEMA) como monómeros adequados para a preparação de MIP com uma composição molar T:M:C (template:monómero:reticulante) de 1:3:20 e 1:2:20, respetivamente, com base nas simulações do SYBYL ${ }^{\mathrm{TM}}$ (Fig. 5).

Os polímeros foram sintetizados em $\mathrm{scCO}_{2}$ usando as proporções preditas anteriormente e o seu desempenho foi avaliado através de testes de afinidade (Fig. 6) que demonstraram ter a mesma tendência das previsões do SYBYL ${ }^{\mathrm{TM}}$.

Os polímeros ITA-MIPs conseguiram remover 2,3 vezes mais ACET do que os polímeros HEMA-MIPs, o que demonstra bem o quanto esta ferramenta computacional pode ser uma mais-valia na previsão de sistemas de afinidade. A abordagem computacional que usa $\mathrm{CO}_{2}$ na simula-

a)

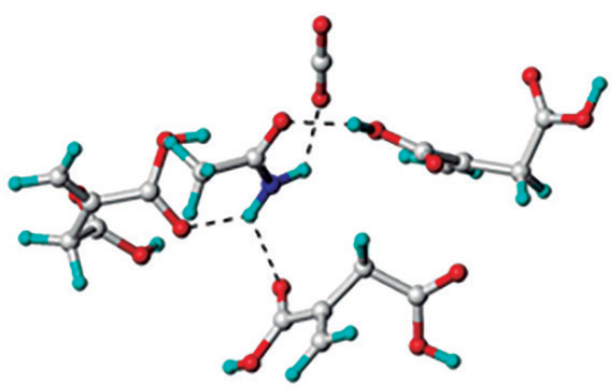

ção é uma maneira acessível, direta e computacionalmente pouco exigente para a otimização da síntese de MIPs utilizando tecnologia supercrítica.

\section{Conclusões}

Os resultados obtidos nos três exemplos mencionados demonstraram um bom desempenho dos materiais desenvolvidos usando tecnologias sustentáveis. Os materiais obtidos são baratos e altamente específicos para aplicações industriais, como a remoção de impurezas do API. Permitem processos de separação muito eficientes e respondem também a um problema ambiental, que é a redução do consumo de solventes orgânicos. Mostrou-se também que o uso de uma abordagem computacional pode ser uma mais-valia no desenho racional dos melhores sistemas de afinidade, evitando métodos complexos e demorados e, consequentemente, reduzindo o tempo e o consumo de solventes orgânicos. A adoção destes métodos contribui para uma mudança de mentalidades, para redesenhar processos e impulsionar o uso de tecnologias verdes no desenvolvimento de materiais de elevada afinidade.

\section{Agradecimentos}

Os trabalhos aqui descritos fazem parte da minha tese de doutoramento, orientada pela Doutora Teresa Casimiro (LAQV-REQUIMTE, FCT-UNL) e coorientada pelo Doutor William Heggie (Hovione), e que foi recentemente distinguida com o 2. ${ }^{\circ}$ prémio "EFCE Excellence Award in Fluid Separations 2018”, pela Federação Europeia de Engenharia Química. Agradeço a todos os que colaboraram

b)

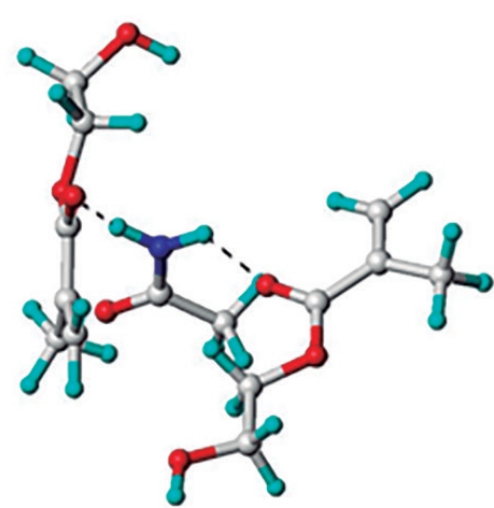

Figura 5 - a) Complexo ACET-ITA-CO ${ }_{2}$, b) Complexo ACET-HEMA-CO 2 . Reproduzida da referência [10] com permissão da Elsevier.
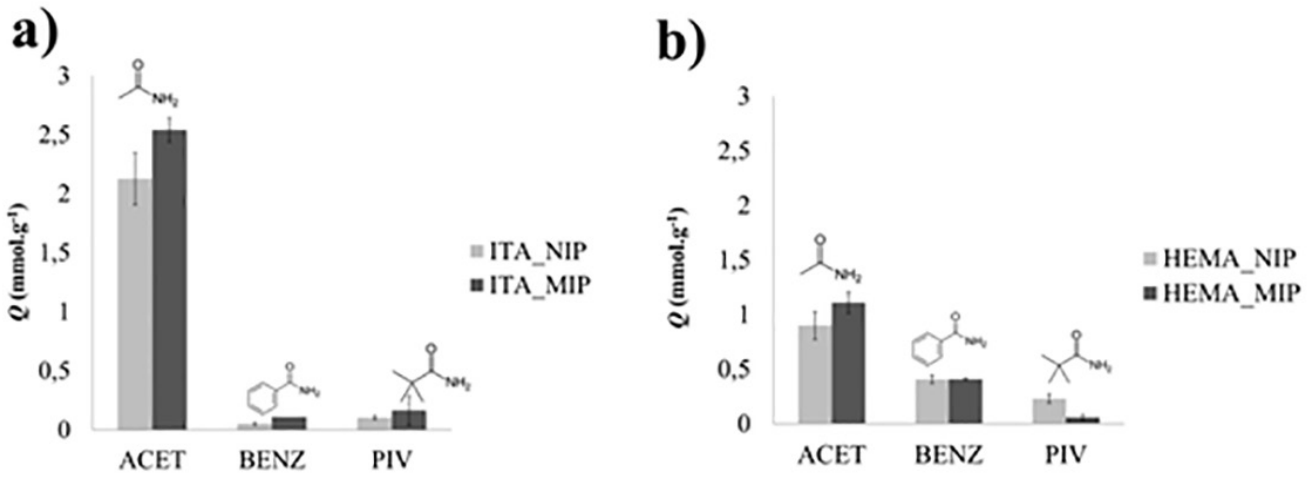

Figura 6 - Ensaios de afinidade em a) ITA-MIPs e b) HEMA-MIPs. Reproduzida da referência [10] com permissão da Elsevier. 
nos trabalhos aqui mostrados, em particular à Maria Inês Lopes (extração de fase sólida - SPE), ao Dr. Francisco M. Dias (funcionalização das partículas de sílica usando plasma, IST), à Dra. Luísa B. Maia (ensaios de EPR), ao Dr. Kal Karim (modulação computacional, Universidade de Leicester, Reino Unido) e ao Prof. Sergey Piletsky (grupo de Biotecnologia da Universidade de Leicester, Reino Unido). O trabalho foi financiado pela Fundação para a Ciência e a Tecnologia (FCT) através do LAQV-REQUIMTE [fundos nacionais - UID/QUI/50006/2013 - cofinanciado pelo FEDER no âmbito do Acordo de Parceria PT2020 (POCI-01-0145-FEDER-007265)] e pelos projetos IF/00915/2014 e PTDC/QEQ-PRS/2757/2012. RV agradece ainda a sua bolsa de doutoramento em empresa SFRH/BDE/51907/2012 financiada pela FCT e pela Hovione FarmaCiência SA.

\section{Referências}

[1] R. Viveiros, T. Casimiro, Química - Bol. SPQ n. ${ }^{\circ} 140$ (2016) 19-26.

[2] I. Chianella, M. Lotierzo, S.A. Piletsky, I.E. Tothill, B. Chen, K. Karim, A.P.F. Turner, Anal. Chem. 74 (2002) 1288-1293.

[3] Y. Hoshino, K.J. Shea, J. Mater. Chem. 21 (2011) 35173521.

[4] R. Viveiros, S. Rebocho, T. Casimiro, Polymers 10 (2018) $1-27$.

[5] L. Müller, R.J. Mauthe, C.M. Riley, M.M. Andino, D. De Antonis, C. Beels, J. DeGeorge, A.G.M. De Knaep, D. Ellison, J.A. Fagerland, R. Frank, B. Fritschel, S. Galloway, E. Harpur, C.D.N. Humfrey, A.S. Jacks, N. Jagota, J. Mackinnon, G. Mohan, D.K. Ness, M.R. O’Donovan, M.D. Smith, G. Vudathala, L. Yotti, Regul. Toxicol. Pharmacol. 44 (2006) 198-211.
[6] K.L. Dobo, N. Greene, M.O. Cyr, S. Caron, W.W. Ku, Regul. Toxicol. Pharmacol. 44 (2006) 282-293.

[7] D. Bartos, S. Gorog, Curr. Pharm. Anal. 4 (2008) 215-230.

[8] C. Wang, X. Hu, P. Guan, D. Wu, L. Qian, J. Li, R. Song, J. Pharm. Biomed. Anal. 102 (2015) 137-143.

[9] R. Viveiros, M.I. Lopes, W. Heggie, T. Casimiro, Chem. Eng. J. 308 (2017) 229-239.

[10] R. Viveiros, K. Karim, S.A. Piletsky, W. Heggie, T. Casimiro, J. Clean. Prod. 168 (2017) 1025-1031.

[11] M. Soares da Silva, R. Viveiros, A. Aguiar-Ricardo, V.D.B. Bonifácio, T. Casimiro, RSC Adv. 2 (2012) 5075-5079.

[12] A.N.C. Martins, S.P. Simeonov, L.M.T. Frija, R. Viveiros, A. Lourenço, M. Soares da Silva, T. Casimiro, C.A.M. Afonso, Ind. Crops Prod. 60 (2014) 226-232.

[13] J.P. Ferreira, R. Viveiros, A. Lourenço, M. Soares da Silva, A. Rosatella, T. Casimiro, C.A.M. Afonso, RSC Adv. 4 (2014) 54948-54952.

[14] M. Soares da Silva, R. Viveiros, P.I. Morgado, A. AguiarRicardo, I.J. Correia, T. Casimiro, Int. J. Pharm. 416 (2011) 61-68.

[15] G. Marcelo, I. Ferreira, R. Viveiros, T. Casimiro, Int. J. Pharm. 542 (2018) 125-131.

[16] A.S. Silva, M.C. Silva, S.P. Miguel, V.D.B. Bonifácio, I.J. Correia, A. Aguiar-Ricardo, RSC Adv. 6 (2016) 3363133635.

[17] A. Ellwanger, C. Berggren, S. Bayoudh, C. Crecenzi, L. Karlsson, P.K. Owens, K. Ensing, P. Cormack, D. Sherrington, B. Sellergren, Analyst 126 (2001) 784-792.

[18] G. Székely, E. Fritz, J. Bandarra, W. Heggie, B. Sellergren, J. Chromatogr. A 1240 (2012) 52-58.

[19] T. Casimiro, R. Viveiros, Patente PT 107208, 2015.

[20] R. Viveiros, F.M. Dias, L.B. Maia, W. Heggie, T. Casimiro, J. Ind. Eng. Chem. 54 (2017) 341-349.

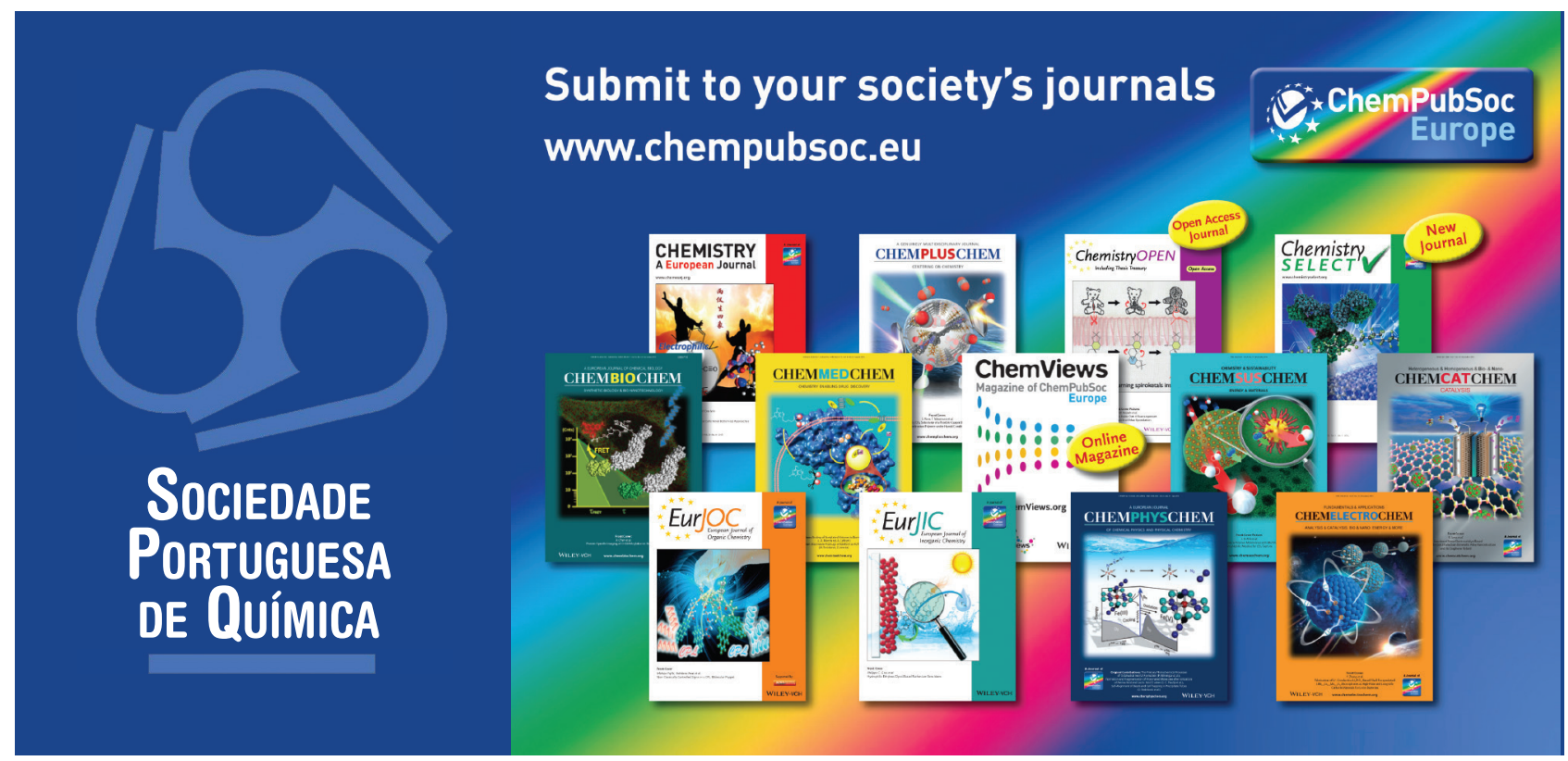

